\title{
Optimizing the Rekeying Cost for Contributory Group Key Agreement Schemes
}

\author{
Wei Yu, Yan (Lindsay) Sun, Member, IEEE, and K.J. Ray Liu, Fellow, IEEE
}

\begin{abstract}
Although a contributory group key agreement is a promising solution to achieve access control in collaborative and dynamic group applications, the existing schemes have not achieved the performance lower bound in terms of time, communication, and computation costs. In this paper, we propose a contributory group key agreement that achieves the performance lower bound by utilizing a novel logical key tree structure, called PFMH, and the concept of phantom user position. In particular, the proposed scheme only needs $O(1)$ rounds of the two-party Diffie-Hellman $(\mathrm{DH})$ upon any single-user join event and $O(\log n)$ rounds of the two-party DH upon any single-user leave event. Both the theoretical bound analysis and simulation show that the proposed scheme achieves a lower rekeying cost than the existing tree-based contributory group key agreement schemes.
\end{abstract}

Index Terms-Security, key management, tree structure.

\section{INTRODUCTION}

$\mathrm{O}$ NE fundamental challenge in securing group applications is to achieve access control such that only authorized group members can access group communications. Group access control is usually achieved by encrypting data using a group key that is shared among all legitimate group members. The issues of establishing and updating group keys are addressed by group key agreement schemes [1], [2], [3], [4], [5], [6], [7], [8], [9], [10], [11], [12], [13], [14], [15], [16], [17], [18], [19], [20], [26], [27]. Since many practical group applications do not prefer utilizing centralized key servers, contributory solutions of key agreement have drawn extensive attention [1], [2], [3], [4], [5], [6], [7], [8], [9], [10], [11], especially for applications where centralized administration and pairwise secure channels are not applicable.

In contributory group key agreement schemes, all group members contribute their shares and compute the group key collaboratively, and the group key is generated as a (usually one-way) function of individual contributions from all group members [1], [2], [3], [5], [4], [6], [7], [8], [9], [10], [11]. Upon membership changes, the group key needs to be updated to incorporate the share from the joining user or to eliminate the share of the leaving user to maintain backward secrecy and forward secrecy [9]. Establishing and updating the group key in large dynamic groups often consumes a considerable amount of computation and communication resources. For large-scale dynamic group

- W. Yu is with Microsoft Corporation, One Microsoft Way, Redmond, WA 98052. E-mail: weiy@microsoft.com.

- Y. Sun is with the Department of Electrical and Computer Engineering, University of Rhode Island, 4 East Alumni Ave., Kingston, RI 02881. E-mail: yansun@ele.uri.edu.

- K.J.R. Liu is with the Department of Electrical and Computer Engineering, University of Maryland, College Park, MD 20742.

E-mail: kjrliu@umd.edu.

Manuscript received 11 Nov. 2004; revised 13 June 2006; accepted 19 Apr. 2007; published online 23 May 2007.

For information on obtaining reprints of this article, please send e-mail to: tdsc@computer.org, and reference IEEECS Log Number TDSC-0162-1104.

Digital Object Identifier no. 10.1109/TDSC.2007.1006. applications where group members do not have ample communication and computation capability, such as in some mobile ad hoc and sensor networks, the bottleneck of utilizing contributory key agreement schemes for access control will be their cost efficiency.

The early design of contributory group key agreement schemes mostly focuses on the efficiency of initial group key establishment, such as in [1], [2], [3]. These schemes, however, encounter a high rekeying cost upon group membership changes. Later, Steiner et al. proposed a family of Group Diffie-Hellman (GDH) protocols by extending the two-party Diffie-Hellman (DH) protocols [21] to the group scenarios [4], [6], [7]. The GDH protocols achieve an efficient key update upon user join but still require a high cost for member leave. Recently, logical key tree structures are used to improve the scalability of contributory key agreements [9], [10]. Kim et al. proposed a tree-based contributory group key agreement protocol called Tree-based Group DiffieHellman (TGDH), where a binary balanced tree is adopted to maintain the keying material [9]. In TGDH, the group key can be updated by performing $\log n$ rounds of the two-party DH upon any single-user join or leave, where $n$ is the group size. Mao et al. proposed another tree-based contributory key agreement scheme called Dynamic SubTree (DST) [10]. By using a special join-tree/exit-tree topology and exploiting cost amortization, DST can reduce the average time cost to $\Theta(\log \log n)$ rounds of the two-party DH for single-user join or leave. However, DST has an unrealistic requirement that members know other members' leave time in advance. When the members' leave time is not known, the time cost upon single-user leave is $\Theta(\log n+\log \log n)$, which is higher than that of TGDH.

What is the lowest possible cost of contributory group key agreement schemes? The theoretical analysis in [22] indicates that for any tree-based contributory group key management scheme, the lower bound of the worst-case cost is $\Theta(\log n)$ rounds of the two-party DH for either user addition or deletion. That is, either the cost for adding a user or the cost for deleting a user is no less than $\Theta(\log n)$. In 
addition, it is obvious that at least one round of the twoparty $\mathrm{DH}$ needs to be performed for adding or deleting a user in any circumstance. Therefore, the lowest possible cost for contributory key agreement is $\Theta(\log n)$ for user join and $O(1)$ for user leave or $\Theta(\log n)$ for user leave and $O(1)$ for user join. Both TGDH and DST do not achieve these lower bounds. In addition, from [22], we can also derive that the total rounds of the two-party $\mathrm{DH}$ for any sequence of $n$ single-user join events and $n$ user leave events is bounded by $\Theta(n \log n)$. This bound is in fact looser than the previous two bounds.

To achieve the lower bound of the rekeying cost, in this paper, we propose a novel and efficient logical key tree structure, called PFMH tree, as well as a cost-minimizing PFMH tree-based contributory group key agreement (PACK) protocol suite that handles dynamic group membership events. The optimality of the proposed PACK protocol suite lies in that it only needs $O(1)$ rounds of the two-party DH upon any single-user join event and $O(\log n)$ rounds of the two-party $\mathrm{DH}$ upon any single-user leave event, which achieves the lower bound. Both theoretical analysis and simulation studies show that PACK has a much lower rekeying cost than the existing tree-based contributory group key agreements.

The rest of this paper is organized as follows: Section 2 briefly introduces security requirements and performance metrics for contributory group key agreement schemes. Section 3 presents the proposed PFMH tree structure, as well as two basic procedures to manage the PFMH key trees. Section 4 describes the proposed PACK protocol suite to optimize the rekeying cost upon single-user join and leave events. Section 5 analyzes the performance of the proposed scheme and demonstrates its efficiency by comparing it with existing tree-based contributory group key agreement schemes through both bound analysis and simulation studies. Section 6 discusses the detection of untruthful users who do not perform the key agreement protocol honestly. Finally, a conclusion is drawn in Section 7.

\section{Security Requirement and Performance METRIC}

In this section, we briefly introduce the security requirements of contributory key agreement, the performance measures, and the implementation cost of the DH protocol between two groups.

Group key management schemes must be able to adjust group secrets subsequent to membership changes, including single-user addition, single-user deletion, group merge, and group partition [9]. Single-user addition (deletion) means that one user joins (leaves) the group. Group merge (partition) involves multiple users who join (leave) the group simultaneously. The security requirements with dynamic membership include group key secrecy, forward secrecy, backward secrecy, and key independence [9]. Group key secrecy, which is the most basic property, requires that it should be computationally infeasible for a passive adversary to discover any group key. Forward secrecy requires that a passive adversary who knows a contiguous subset of old group keys cannot discover subsequent group keys, whereas backward secrecy requires that a passive adversary who knows a contiguous subset of group keys cannot discover preceding group keys. Key independence, which is the strongest property, requires that a passive adversary who knows a proper subset of group keys cannot discover any other group key. According to [9], key independence can be achieved when both forward secrecy and backward secrecy are achieved.

The overhead of group key agreement involves the computation cost, communication cost, and time cost. Since most of the existing contributory key agreement schemes use the two-party $\mathrm{DH}$ protocol [21] as a basic building module, the computation cost comes mainly from the cryptographic primitives that are needed to perform the two-party $\mathrm{DH}$, such as modular exponentiation, and the communication cost comes from sending and receiving rekeying messages. The time cost is used to describe the latency in group key establishing and updating. In contributory group key agreement, by exploiting the possible parallelism when performing group key establishing and updating, the time cost can be significantly reduced.

Among existing contributory group key agreement schemes [1], [2], [3], [4], [5], [6], [7], [8], [9], [10], [11], the tree-based schemes are the most promising because of their scalability. Next, we introduce the implementation of the two-group DH (two-party DH among two groups), which is the basic building module for most tree-based contributory group key agreement schemes. Let $\mathbf{A}$ and $\mathbf{B}$ denote two subgroups, where the users in $\mathbf{A}$ share a common group key $K_{A}$, and the users in $\mathbf{B}$ share a common group key $K_{B}$. Let $f(K)$ (which we refer to as the blinded key of key $K$ ) denote the modular exponentiation operation, that is,

$$
f(K)=g^{K} \bmod p,
$$

where $g$ is the exponential base and $p$ is the modular base. The two-group DH can be implemented as follows: Each subgroup elects one member as its delegate, which will compute and send its blinded subgroup key to all members of the other subgroup. Suppose that member $A_{1}$ is the delegate elected by the subgroup $\mathbf{A}$ and member $B_{1}$ is the delegate elected by the subgroup $\mathbf{B}$. To perform the twogroup $\mathrm{DH}$ between these two subgroups, $A_{1}$ and $B_{1}$ need to exchange the following keying messages: $A_{1}$ sends the blinded key $f\left(K_{A}\right)$ to all members of subgroup $\mathbf{B}$, and $B_{1}$ sends the blinded key $f\left(K_{B}\right)$ to all members of subgroup $\mathbf{A}$. Now, each member in $\mathbf{A}$ or $\mathbf{B}$ then calculates the new group key $K_{A B}$ as follows:

$$
K_{A B}=\left(f\left(K_{B}\right)\right)^{K_{A}} \bmod p=\left(f\left(K_{A}\right)\right)^{K_{B}} \bmod p .
$$

In this implementation, each member needs at least one modular exponentiation operation to calculate the new group key. If a delegate does not know its own subgroup's blinded key, one extra modular exponentiation operation is also needed to calculate the blinded key. For the communication cost, each delegate needs to send a keying message to all the members in the other subgroup. In this paper, we use $C_{\text {cast }}(n, \ell)$ to denote the communication cost needed to send a message with length $\ell$ to $n$ nodes and use $C_{m e}$ to denote the computation cost of a modular exponentiation operation. Thus, for each round of the twogroup $\mathrm{DH}$ with the size of subgroups being $n_{1}$ and $n_{2}$ and 
the keying message length being $\ell$, the communication cost is $C_{\text {cast }}\left(n_{1}, \ell\right)+C_{\text {cast }}\left(n_{2}, \ell\right)$, and the computation cost is no more than $\left(n_{1}+n_{2}+2\right) C_{m e}$.

It is worth noting that sending a message to $n$ nodes can be implemented in many ways. It can either be implemented through multicast communications, which we refer to as multicast- $n$, or be implemented through unicast, which we refer to as unicast- $n$. In general, the communication cost of a multicast- $n$ operation is not the same as the communication cost of a unicast- $n$ operation. The former usually incurs less communication cost than the latter. Further, the gap between the communication cost of a multicast- $n$ operation and the communication cost of a unicast- $n$ operation may vary according to the underlying network architectures. For example, in wireless networks, the gap is usually very obvious due to the broadcast nature of wireless media, whereas in wired networks without link-level multicast support, the gap is usually not that obvious.

In this paper, when analyzing the communication cost of sending a message to $n$ nodes, both terms (multicast- $n$ and unicast- $n$ ) will be used. Although the communication cost of multicast- $n_{1}$ and multicast- $n_{2}$ with $n_{1} \neq n_{2}$ are usually different, to simplify our illustration, in this paper, we will not distinguish them. Let $C_{\text {multicast }}(\ell)$ denote the communication cost of a multicast- $n$ operation, and let $C_{\text {unicast }}(\ell)$ denote the communication cost of a unicast- 1 operation, where $\ell$ is the length of the message to be sent. Further, when performing the two-group DH between two subgroups, the only messages exchanged are their blinded keys. Since, in general, all blinded keys have the same length, without loss of generality, the message length $\ell$ will not be explicitly stated. Besides exchanging blinded keys, a user may also need to send messages to all of the group members when it wants to join or leave a group. In this paper, we use $C_{\text {broadcast }}(\ell)$ to denote the communication cost incurred by broadcasting a message with length $\ell$ to all group members.

\section{Pfmh Key Tree Structure and Basic Procedures}

In tree-based contributory group key agreement schemes, keys are organized in a logical tree structure, referred to as the key tree. In a key tree, the root node represents the group key, leaf nodes represent the members' private keys, and each intermediate node corresponds to a subgroup key shared by all the members (leaf nodes) under this node. The key of each nonleaf node is generated by performing the two-party DH between the two subgroups represented by its two children where each child represents the subgroup including all the members (leaf nodes) under this node [9]. Since the two-group DH is used, the key tree is binary. For each node in the key tree, the key path denotes the path from this node to the root, and the copath denotes the sequence of siblings of each node on its key path. Fig. 1 shows a simple key tree example with six members, where $M_{i}$ denotes the $i$ th group member, and $(l, v)$ denotes the $v$ th node at level $l$ of the tree. For example, for member $M_{2}$, its key path is the sequence of nodes $\{(3,1),(2,0),(1,0),(0,0)\}$, and its copath is the sequence of nodes $\{(3,0),(2,1),(1,1)\}$.

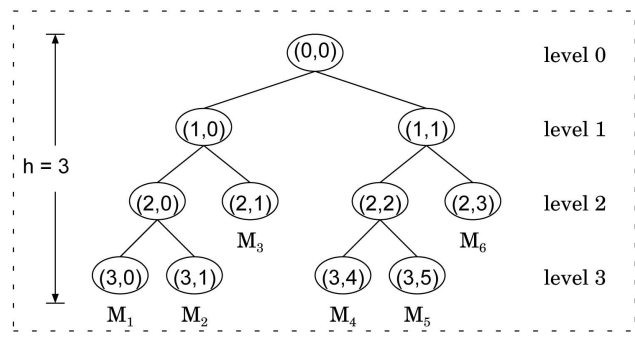

Fig. 1. A simple key tree example.

According to [9], in order to compute the group key, a node only needs to know its own key and all the blinded keys on its copath. In other words, for a node to be able to calculate the group key, it only needs to know its own keys and all the blinded keys on its copath. For example, as shown in Fig. $1, M_{2}$ only needs to know its own key and the blinded keys represented by the nodes $(3,0),(2,1)$, and $(1,1)$ in order to calculate the group key.

A leaving user can leave from an arbitrary position in the key tree. In fact, for user leave, when group members have similar computation and communication capability, the best tree structure that reduces the worst-case rekeying overhead is a balanced key tree structure. ${ }^{1}$ When using a balanced key tree structure, as in TGDH [9], the worst-case rekeying time cost for both user leave and user join is $O(\log n)$. In order to further reduce the rekeying time cost for user join, one way is to always insert the joining user at the root of the key tree and, consequently, the rekeying time cost for single-user join becomes $O(1)$. However, such a scheme may result in an extremely unbalanced key tree structure and increase the rekeying cost for user leave to $O(n)$.

In order to achieve the lower bound for both user join and user leave simultaneously, in this paper, we propose a novel and efficient key tree structure for contributory group key agreement schemes, which we refer to as the PFMH tree. PFMH tree is a combination of two special key tree structures: partially full (PF) key tree and maximum height $(\mathrm{MH})$ key tree. In this paper, the size of a key tree is defined as the total number of leaf nodes in this tree, the function $\log ()$ and $\log _{2}()$ will be used exchangeably, and when we say a "full (key) tree," we always mean a fully balanced binary (key) tree with size $2^{k}$, where $k$ is a nonnegative integer.

Definition 1 (PF key tree). Let $T$ be a binary key tree of size $n$, and let $n^{\prime}=2^{\lfloor\log n\rfloor}$. T is a PF key tree if and only if it satisfies one of the following properties: 1) $T$ is a full key tree, and 2) the left subtree of $T$ is a full key tree with size $n^{\prime}$, and the right subtree of $T$ is a PF key tree with size $\left(n-n^{\prime}\right)$.

Definition 2 (MH key tree). A key tree $T$ of size $n$ is a MH key tree if and only if it satisfies one of the following properties: 1) $n=1$, and $T$ is a tree with only one leaf node. 2) The right subtree of $T$ is a leaf node, and the left subtree of $T$ is an MH key tree with size $n-1$.

Definition 3 (PFMH key tree). A key tree $T$ of size $n$ is a PFMH key tree if and only if it satisfies one of the following

1. The case where users have varying computation and communication capabilities is beyond the scope of this paper. 


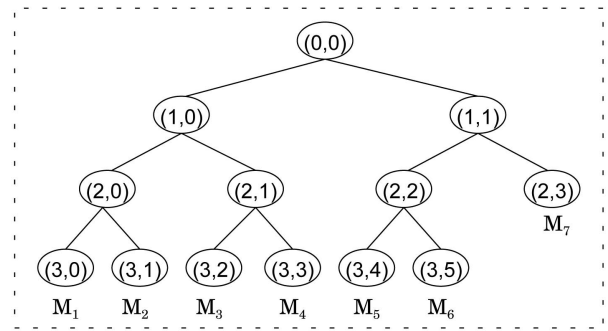

(a)

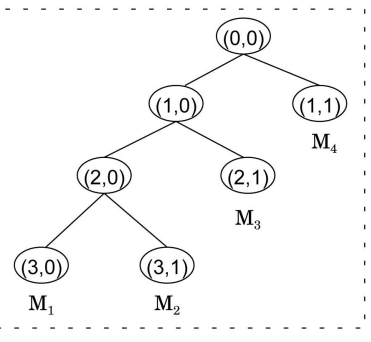

(b)

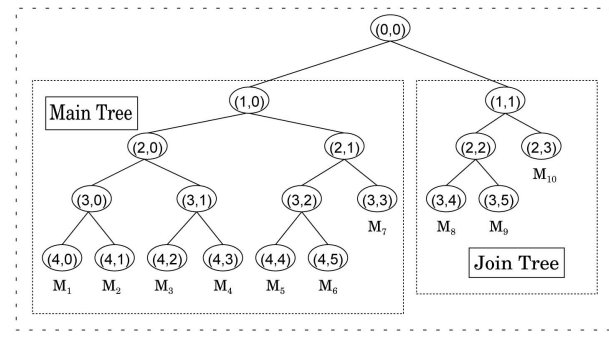

(c)

Fig. 2. Examples of (a) PF, (b) MH, and (c) PFMH key trees.

properties: 1) $T$ is a PF key tree. 2) The left subtree of $T$ is a PF tree, and the right subtree of $T$ is an $M H$ tree.

According to the above definitions, we can see that the height of a PF key tree with size $n$ is $\lceil\log n\rceil$, the height of an MH tree with size $n$ is $n-1$. In this paper, without introducing ambiguity, we will use $\lceil\log n\rceil$ and $\log n$ exchangeably. Also, given a PFMH key tree $T$, we will use main tree to refer to the PF subtree of $T$, denoted by $T_{\text {main }}$, and use join tree to refer to the $\mathrm{MH}$ subtree of $T$, denoted by $T_{\text {join }}$. It is easy to see that the height of $T_{\text {main }}$ is always bounded by $\log n$. Fig. 2 illustrates these special key tree structures. Next, we describe two basic procedures to manage and update PFMH key trees: unite and split.

Let $\mathcal{T}=\left\{T_{1}, \ldots, T_{L}\right\}$ be a set of full key trees. Each key tree $T_{i} \in \mathcal{T}$ represents a subgroup, and each leaf node of $T_{i}$ is a member of this subgroup. If a group member belongs to $T_{i}$ and $T_{i} \in \mathcal{T}$, then this group member belongs to $\mathcal{T}$. The procedure unite $(\mathcal{T})$ is to combine those key trees in $\mathcal{T}$ into a single PF key tree through performing a series of two-group DHs among these subgroups, as well as the subgroups generated during this procedure. In general, given a set of full key trees $\mathcal{T}$, the result of unite $(\mathcal{T})$ may not be unique, but all of the obtained PF key trees have a similar structure. In this paper, we consider a special case where the full key trees in $\mathcal{T}$ are ordered and indexed according to their sizes. Moreover, any group member in $\mathcal{T}$ knows the indices and sizes of any trees in $\mathcal{T}$, as well as the structure of these trees. The structure of a tree refers to the list of group members belonging to this tree and their exact positions in this tree. Then, a group member can decide with whom it should perform the two-group $\mathrm{DH}$ and in what order.

Procedure 1 presents one specific implementation of unite $(\mathcal{T})$ for this special case. According to Procedure 1 , the whole procedure is partitioned into many rounds. At the beginning of each round, there remains a set of full trees (subgroups) indexed according to their sizes and their subtrees' indices in the previous rounds. The larger the size of a subgroup, the lower its index. In each round, a remaining subgroup may either remain alone or be paired with another remaining subgroup according to the following rule: Two subgroups, $T_{i}$ and $T_{j}(i<j)$, will be paired together if and only if all of the three conditions can be satisfied:

- There is no other remaining subgroup in the round with an index lying between $i$ and $j$.
- The total number of subgroups with a size equal to $\left|T_{i}\right|$ and with an index lying before $T_{i}$ is even.

- $\left|T_{i}\right|=\left|T_{j}\right|$ or $T_{j}$ is the subgroup with the largest index.

It is easy to see that in each round, a subgroup will either remain alone or be paired with one and only one other subgroup to build a larger subgroup. Further, in each round, all pairs of subgroups can perform the two-group $\mathrm{DH}$ between them in parallel, which can significantly reduce the time cost. If Procedure 1 is followed by all group members, the obtained PF key tree is unique, and each member can know its location in the final PF before starting the procedure and can locally construct the final PF tree without explicitly exchanging key tree updating information.

Procedure 1 unite $\left(\left\{T_{1}, \ldots, T_{L}\right\}\right)$ $\triangleright \mathcal{T}=\left\{T_{1}, \ldots, T_{L}\right\} ;\left|T_{i}\right| \geq\left|T_{j}\right|$ for any $1 \leq i<j \leq L$; each member in $T_{i} \in \mathcal{T}$ knows the index and size of any tree $T_{j} \in \mathcal{T}$, as well as the structure $T_{j}$, including the list of group members in $T_{j}$ and their exact positions in $T_{j}$.

$\mathcal{T}^{\prime}=\mathcal{T} ; L^{\prime}=L ;$

while $\left(\left|\mathcal{T}^{\prime}\right|>1\right)$ do

$/{ }^{*}$ Executed in parallel:*/

for (each pair of trees $T_{i}, T_{i+1} \in \mathcal{T}^{\prime}$ ) do

if ((the total number of trees in $\mathcal{T}^{\prime}$ with size equal to

$\left|T_{i}\right|$ and with index before $T_{i}$ is even) AND

$\left(\left|T_{i}\right|=\left|T_{i+1}\right|\right.$ or $T_{i+1}$ is the tree in $\mathcal{T}^{\prime}$ whose index is the largest)) then

Two delegates will be elected by subgroups $T_{i}$ and $T_{i+1}$ to perform the two-group $\mathrm{DH}$ between them, and a new group key $K$ will be generated. A new key tree will be generated with its root node representing $K$, with the left child of the root node being $T_{i}$ and with the right child of the root node being $T_{i+1}$. Remove $T_{i}$ and $T_{i+1}$ from $\mathcal{T}^{\prime}$. end if

end for

Put all newly generated key trees in this round into $\mathcal{T}^{\prime}$, and let $L^{\prime}$ be the total number of key trees now in $\mathcal{T}^{\prime}$. Re-index all the key trees in $\mathcal{T}^{\prime}$ with integers ranging from 1 to $L$ in such a way that a tree is assigned index $i$ (that is, this tree's name becomes $T_{i}$ ) if and only if: 1 ) for any tree $T_{j} \in \mathcal{T}^{\prime}$ with index $j<i$, all subtrees of $T_{j}$ that directly come from $\mathcal{T}$ have lower indices than all 


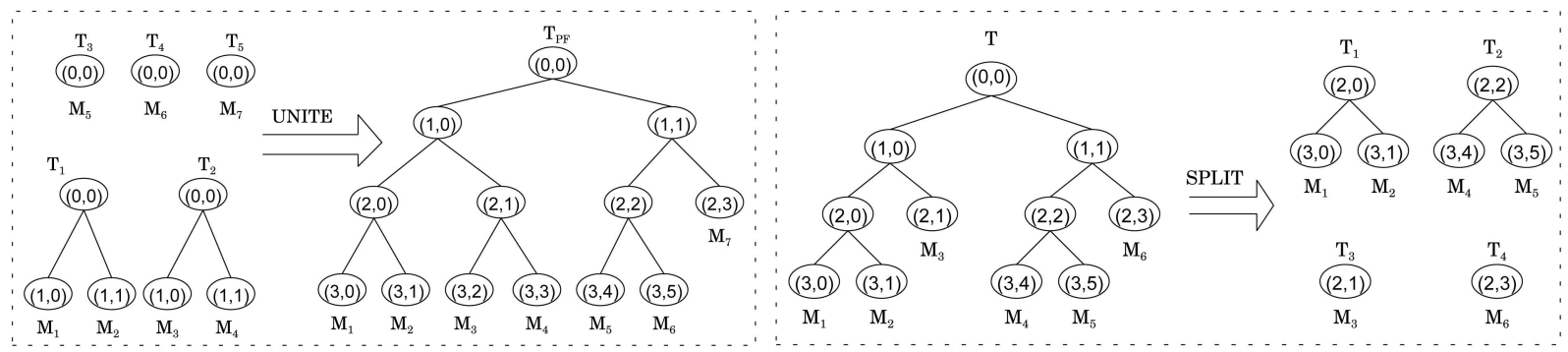

Fig. 3. Examples of a key tree update after applying (a) unite and (b) split procedures.

subtrees of $T_{i}$ that directly come from $\mathcal{T}$; and 2) for any tree $T_{j} \in \mathcal{T}^{\prime}$ with $j>i$, all subtrees of $T_{j}$ that directly come from $\mathcal{T}$ have higher indices than all subtrees of $T_{i}$ that directly come from $\mathcal{T}$.

\section{end while}

Return the remaining tree $T_{1}$ in $\mathcal{T}^{\prime}$, which is the final PF key tree. Meanwhile, each member in the final PF key tree will construct the final key tree structure locally by following the above key tree generation procedure.

Given a key tree $T$, the procedure $\operatorname{split}(T)$ is to partition $T$ into a set of full key trees with the minimum set size. Specifically, after applying the procedure $\operatorname{split}(T)$, any obtained key tree is a full key tree, and no more than one obtained key tree comes from any full subtree of $T$. Procedure 2 presents a way to locally and virtually split a key tree, where "locally" means that no intercommunication is needed among group members and each member only needs to update the key tree structure maintained by itself locally, whereas "virtually" means that no two-group DH is needed to perform "split." Meanwhile, the set of obtained full key trees are also indexed according to their size and their positions in the original key tree.

Procedure $2 \operatorname{split}(T)$

if ( $T$ is a full tree) then

Return $\{T\}$;

else if ( $T$ is empty) then

Return $\emptyset$.

\section{else}

Let $T_{\text {left }}$ and $T_{\text {right }}$ be the left and right subtrees of $T$; Return $\operatorname{split}\left(T_{\text {left }}\right) \bigcup \operatorname{split}\left(T_{\text {right }}\right)$.

\section{end if}

Let $L$ be the number of obtained full key trees. Index these key trees with the integers ranging from 1 to $L$ in such a way that a tree is indexed as $T_{i}$ if and only if: 1) for any tree $T_{j} \in \mathcal{T}^{\prime}$ with $j<i,\left|T_{j}\right|>\left|T_{i}\right|$ or $T_{j}$ lies in the left side of $T_{i}$ in $T$; and 2) for any tree $T_{j} \in \mathcal{T}^{\prime}$ with $j>i$, $\left|T_{j}\right|<\left|T_{i}\right|$ or $T_{j}$ lies in the right side of $T_{i}$ in $T$.

Fig. 3 shows two examples of a key tree update when applying unite and split procedures. Fig. 3a demonstrates how the key tree is updated when five full key trees are united into a PF key tree. Fig. 3b demonstrates how the key trees are updated when a PFMH key tree is split into a set of full key trees.

In the split procedure, each group member (leaf node) only needs to truncate the current key tree maintained by itself, so no communication cost and negligible computation and time costs are needed. In the unite procedure, an extra cost will be incurred when performing a sequence of twogroup DHs to generate the new key tree. Next, we analyze the cost associated with the unite procedure described in Procedure 1. The results will be used later to analyze the cost of those proposed key agreement protocols.

Theorem 1. Let $\mathcal{T}=\left\{T_{1}, \ldots, T_{L}\right\}$ be a set of full key trees, with $\sum_{i=1}^{L}\left|T_{i}\right|=n,\left|T_{1}\right| \geq\left|T_{2}\right| \geq \ldots \geq\left|T_{L}\right|$, and the subscript $\ell$ being the index of the full tree $T_{\ell}$. Assume that any group member in any full tree $T_{i} \in \mathcal{T}$ knows the index and size of any tree $T_{j} \in \mathcal{T}$, as well as the structure of $T_{j}$. Then, the costs associated with the unite $(\mathcal{T})$ using Procedure 1 can be bounded as follows:

1. The time cost, which is the number of parallel rounds that needs to executed, is upper bounded by $\log n$ in all situations.

2. The total communication cost is upper bounded by $2(L-1) C_{\text {multicast }}$ in all situations provided that the exchange of keying materials between two subgroups during performing the two-group $\mathrm{DH}$ is implemented using multicast.

3. Consider the special situation that $\left|T_{i}\right|=1$ for all $1 \leq i \leq L$, the total computation cost is upper bounded by $n(\log n+2) C_{m e}$, and the total communication cost is upper bounded by $(n \log n) C_{\text {unicast }}$ provided that the exchange of keying materials between two subgroups during performing the two-group DH is implemented using unicast.

4. Consider the special situation that $\left|T_{i}\right| \neq\left|T_{j}\right|$ for any $1 \leq i \neq j \leq L$, the total computation cost is upper bounded by $2(n+\log n) C_{m e}$, and the total communication cost is upper bounded by $2 n C_{\text {unicast }}$ provided that the exchange of keying materials between two subgroups during performing the two-group $D H$ is implemented using unicast.

5. Consider the special situation that $\left|T_{1}\right| \geq n / 2$ and for any tree $T_{i} \in \mathcal{T}$, there exists no more than one other tree in $\mathcal{T}$ with the same size as $T_{i}$, the total computation cost is upper bounded by $(2.5 n+2 L) C_{m e}$, and the total communication cost is upper bounded by $2.5 n C_{\text {unicast }}$ provided that the exchange of keying materials between two subgroups during performing the two-group DH is implemented using unicast.

6. Consider the special situation where $\left|T_{1}\right|<n / 2$ and for each tree $T_{i} \in \mathcal{T}$ there exists no more than one other tree in $\mathcal{T}$ with the same size as $T_{i}$, the total computation cost is upper bounded by $(3 n+2 L) C_{m e}$, and the total 
communication cost is upper bounded by $3 n C_{\text {unicast }}$ provided that the exchange of keying materials between two subgroups during performing the two-group $\mathrm{DH}$ is implemented using unicast.

Proof. See Appendix.

\section{PACK: A PFMH TREe-Based Contributory Group Key AGREemENT}

In this section, we describe the proposed PACK protocol suite. As a contributory scheme, in PACK, each group member equally contributes its share to the group key, and this share is never revealed to the others. To satisfy the security requirements, PACK includes a set of rekeying protocols to update the group key upon group membership change events. Compared with the existing tree-based contributory group key agreement schemes, PACK can achieve the minimum rekeying time cost upon membership change events in the sense that for any single-user join event, the rekeying time cost is of order $O(1)$, and for any single-user leave event, the rekeying time cost is of order $O(\log n)$. Meanwhile, the rekeying computation and communication costs can also be significantly reduced compared with the existing tree-based contributory group key agreement schemes. This is achieved through adopting the proposed PFMH tree as the underlying key tree structure and introducing phantom nodes in the key tree to handle member leave.

In PACK, each member will maintain and update the global key tree locally. Each group member knows all the subgroup keys on its key path and knows the ID and the exact location of any other current group member in the key tree. As to be shown next, upon a group membership change event, a group member only needs to update the global key tree maintained by itself, which can greatly reduce the communication overhead. In PACK, when a new user joins the group, it will always be attached to the root of the join tree to achieve $O(1)$ rekeying cost in terms of computation per user, time, and communication. When a user leaves the current group, according to the leaving member's location in the key tree, as well as whether this member has a phantom location in the key tree, different procedures will be applied, and the basic idea is to update the group key in $O(\log n)$ rounds and simultaneously reduce the communication and computation costs.

\subsection{Single-User Join Protocol}

When a prospective user $M$ wants to join the group $\mathcal{G}$, it initiates the single-user join protocol by broadcasting a request message that contains its member ID, a join request, its own blinded key, some necessary authentication information, and its signature for this request message. After receiving this user join request message, the current group members will check whether $M$ has the privilege to join the group based on certain group access control policies. If $M$ has the authorization to join, the key tree will be updated by incorporating $M^{\prime}$ 's share, and a new group key will be generated in order to incorporate a secret share from $M$ and to guarantee the group keys' backward secrecy. Procedure 3 describes the single-user join protocol in PACK.

Procedure $3 \operatorname{join}(\mathcal{G}, M)$

$\triangleright T$ is the PFMH key tree of group $\mathcal{G}, T_{\text {main }}$ is the main tree of $T, T_{\text {join }}$ is the join tree of $T$.

if $\left(T_{\text {join }}\right.$ is empty) then

A delegate will be elected by group $\mathcal{G}$ to perform the two-group DH with $M$, and a new group key $K$ will be generated. A leaf node will be created to represent $M$, and a new root node will be created to represent $K$ with its right child being the node representing $M$ and its left child being $T_{\text {main }}$. The node representing $M$ becomes the join tree of the updated key tree. else

Round 1: A delegate will be elected by group $T_{\text {join }}$ to perform the two-group $\mathrm{DH}$ with $M$, and a new subgroup key $K_{\text {join }}$ will be generated. A leaf node will be created to represent $M$, and a new intermediate node will be created for $K_{\text {join }}$ with its right child being $M$ and its left child being the old $T_{\text {join }}$.

Round 2: Two delegates will be elected separately by $T_{\text {main }}$ and the new join tree to perform two-group $\mathrm{DH}$ between them, and a new group key $K$ will be generated. A new root node will be created to represent $K$ with its right child being $T_{\text {join }}$ and its left child being $T_{\text {main }}$.

\section{end if}

Each current member updates the key tree maintained by itself locally according to the above key tree update procedure, and a delegate will send an updated copy of the key tree to the new joining member $M$.

In PACK, the rekeying upon single-user join needs to perform at most two rounds of the two-group $\mathrm{DH}$. If the join tree is not empty, a new join tree is generated by performing the two-group $\mathrm{DH}$ between the new member and the old join tree, with the left subtree being the old join tree and the right subtree being the node representing the new member. If the join tree is empty, the node representing the new member becomes the join tree. The group key is generated by performing the two-group $\mathrm{DH}$ between the new join tree and the main tree. Since all the current members know the group key tree structure and know the location that the new member should be put in, they can update the key tree themselves.

Fig. 4 shows two examples of a key tree update upon single-user join events. In the first example, the join tree is empty, and the main tree consists of four members. After the new member $M_{5}$ joins the group, a new node is created to act as the new root, and the node $(1,1)$ becomes the new join tree that represents $M_{5}$. In the second example, when $M_{6}$ joins the group, at the first round, the two-group $\mathrm{DH}$ is first performed between $M_{5}$ and $M_{6}$ to generate a new join tree, at the second round, the two-group DH is performed between the new join tree and the main tree to generate a new group key.

Table 1 lists the rekeying cost upon a single-user join event in PACK, where $n$ denotes the total number of leaf 


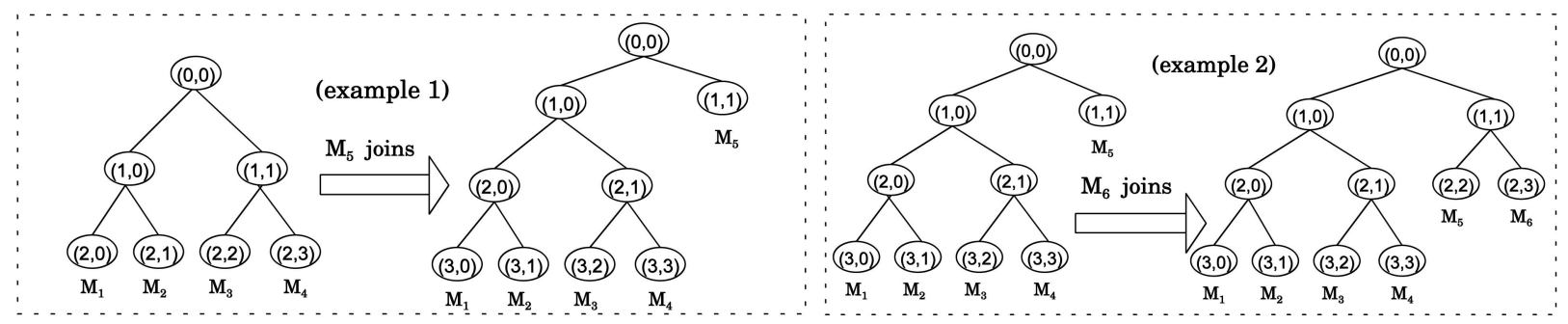

Fig. 4. Examples of a key tree update upon a single-user join event.

TABLE 1

Rekeying Cost upon a Single-User Join Event

\begin{tabular}{|c|c|c|c|c|}
\hline & time cost & $\begin{array}{c}\text { communication cost } \\
\text { in term of multicast }\end{array}$ & $\begin{array}{c}\text { communication cost } \\
\text { in term of unicast }\end{array}$ & computation cost \\
\hline case 1 & 1 & $2 C_{\text {multicast }}$ & $n C_{\text {unicast }}$ & $(n+2) C_{m e}$ \\
\hline case 2 & 2 & $4 C_{\text {multicast }}$ & $\left(n+\left|T_{\text {join }}\right|+1\right) C_{\text {unicast }}$ & $\left(n+\left|T_{\text {join }}\right|+3\right) C_{m e}$ \\
\hline
\end{tabular}

nodes in the new group, and $\left|T_{j o i n}\right|$ is the old join tree size. Case 1 considers the situation that the join tree is empty, and the protocol only needs to perform one round of the two-group DH. Case 2 considers the situation that the join tree is not empty, and the protocol needs to perform two rounds of the two-group DH. For Case 2, the term $\left|T_{\text {join }}\right|+2$ in the computation cost comes from performing the twogroup DH between the new member and the old join tree. Since in general $\left|T_{\text {join }}\right| \ll n$, this term usually can be ignored.

It is worth pointing out that when we calculate the time complexity, we have not considered the extra time needed for the join user to tell the group that it wants to join. However, this does not affect our results because in our time complexity analysis, we use the "round" as the unit. In other words, we do not strictly require the two messages' exchange to be synchronized. Instead, how this can be implemented really depends on the specific implementation of the two-group DH.

\subsection{Single-User Leave Protocol}

When a current group member $M$ wants to leave the group, it broadcasts a leave request message to initiate the singleuser leave protocol, which contains its ID, a leave request, and a signature for this message. Once $M$ leaves the group, the group key will be updated to remove M's share, and all the keys on $M$ 's key path will be updated to maintain group keys' forward secrecy. In PACK, to reduce the rekeying cost upon a single-user leave event, we introduce the concept of a phantom node that allows an existing member to simultaneously occupy more than one leaf node in the key tree. In particular, when member $M$ leaves the group, another group member $M^{\prime}$ will move to the position occupied by $M$ in the key tree and generate a new secret key, and all the keys on $M$ 's key path will be recursively updated. It is worth noting that here "moving" only means that each member adjusts the location of $M^{\prime}$ and $M$ in the key tree. After moving $M^{\prime}$ to $M^{\prime}$ 's position, the node that $M^{\prime}$ previously occupied will not be deleted immediately. As a result, now $M^{\prime}$ occupies two leaf nodes in the key tree. We refer to the node associated to $M^{\prime \prime}$ s previous position as the phantom node, which is known by all group members. In order to maintain the group keys' forward secrecy, a phantom node should be deleted no later than the associated group member leaving the group. Procedure 4 describes the single-user leave protocol in PACK.

Procedure 4 Leave $(\mathcal{G}, M)$

$\triangleright T$ is the PFMH key tree of $\mathcal{G}$, and $n$ is the size of $T, T_{\text {main }}$, and $T_{\text {join }}$ are the main tree and join tree of $T$.

if $\left(\left(M \in T_{\text {join }}\right)\right.$ AND $\left.\left(1<\left|T_{\text {join }}\right| \leq \log n\right)\right)$ then

SCENARIO 1: Let $\mathrm{P}$ be M's sibling, remove $\mathrm{M}$ and M's parent from the key tree. If $\mathrm{P}$ has no children, change $\mathrm{P}^{\prime}$ s secret share; otherwise, change $\mathrm{P}^{\prime}$ 's right child's secret share. Recursively update all the keys on P's key path by applying multiple rounds of the two-group DH.

else if $\left(\left(M \in T_{j o i n} \operatorname{AND}\left(\left|T_{j o i n}\right|=1\right.\right.\right.$ OR $\left.\left.\left|T_{j o i n}\right|>\log n\right)\right)$ OR $\left(M \in T_{\text {main }}\right.$ AND $\left.\left|T_{\text {join }}\right|>1\right)$

OR ( $M \in T_{\text {main }}$ AND $M$ is the rightmost nonphantom leaf node)

OR $\left(M \in T_{\text {main }}\right.$ AND $M$ has a phantom node in $\left.\left.T\right)\right)$ then

SCENARIO 2: First, remove all phantom nodes and $M$ from $T$. Second, apply the split procedure, and let $\mathcal{T}=\left\{T_{1}, \ldots, T_{L}\right\}=\operatorname{split}(T)$. Third, change $T_{L}$ 's rightmost leaf node's secret share and recursively update all the subgroup keys on this left node's key path in $T_{L}$. Fourth, apply the unite procedure unite $(\mathcal{T})$.

else

SCENARIO 3: Find the rightmost nonphantom leaf node $M^{\prime}$ in $T$. Let $P_{\text {new }}$ denote the node occupied by $M$ and $P_{\text {old }}$ denote the node occupied by $M^{\prime} . M^{\prime}$ moves to $P_{\text {new }}$ and generates a new secret share for this location. If $P_{\text {old }}$ lies in the join tree, then remove 


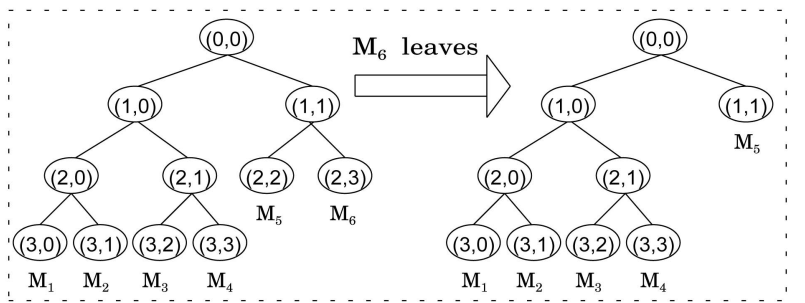

Fig. 5. An example of a key tree update upon single-user leave under the first scenario.

$P_{\text {old }}$ and the root of $T$; otherwise, let $P_{\text {old }}$ be $M^{\prime \prime}$ s phantom node. Recursively update all the keys on $P_{\text {new }}$ 's key path by applying multiple rounds of the end if two-group DH.

All members update the key tree maintained by them locally according to the above key tree update procedure.

SCENARIO 1. This scenario considers the case that the leaving member $\mathrm{M}$ is in the join tree, and the size of the join tree is no larger than $\log n$. In this case, since the depth of the join tree is no more than $\log n$, we can simply remove $M$ 's share from the group key by removing $M$ from the key tree, changing one current member's secret share (which member's share should be changed is described in Protocol 4) and recursively updating all the keys on M's key path. Meanwhile, all members update the key tree maintained by themselves.

Let $h$ be M's depth in $T$. Since at most $h-1$ rounds of two-group DH protocols need to be performed recursively, the time cost is upper bounded by $h-1$. Except the last round, which involves all the existing members, in the $i$ th $(1 \leq i<h-1)$ round, at most $\left|T_{\text {join }}\right|-h+i+1$ members are involved. Then, the total computation cost is upper bounded by $\left(n+h-1+\sum_{k=\left|T_{\text {jin }}\right|-h+2}^{\left|T_{j a i n}\right|-1} k\right) C_{m e}$, where $n$ comes from the last round, $h-1$ comes from the number of blinded keys that needs to be calculated, and $\left|T_{\text {join }}\right|-h+1+i$ comes from the $i$ th round. Since $\left|T_{\text {join }}\right| \leq \log n$, a loose upper bound is $\left(n+\sum_{k=1}^{\log n} k\right) C_{m e}$ or $\left(n+0.5(\log n)^{2}\right) C_{m e}$. Similarly, it is easy to check that the total communication cost in terms of multicast is upper bounded by $2(h-1) C_{\text {multicast, }}$ and the total communication cost in terms of unicast is upper bounded by $\left(n+0.5(\log n)^{2}\right) C_{\text {unicast }}$.

Fig. 5 shows one example of a key tree update upon single-user leave under this scenario. In this example, user $M_{6}$ leaves the group where node $(1,0)$ is the root of the main tree and node $(1,1)$ is the root of the join tree. Since the size of the join tree is 2, according to Procedure 4, the node representing $M_{6}$ will be directly removed from the key tree, $M_{5}$ changes its secret share, and a new group key will be generated by applying the two-group DH between $M_{5}$ and the subgroup in the main tree.

SCENARIO 2. This scenario considers the case that any of the following situations happens:

1. The leaving member $\mathrm{M}$ is in the join tree, and the size of the join tree is either larger than $\log n$ or equal to 1.

2. $\mathrm{M}$ is in the main tree, and the size of the join tree is larger than 1 .
3. $\mathrm{M}$ is in the main tree and is the rightmost nonphantom leaf node.

4. $\mathrm{M}$ is in the main tree and occupies a phantom node in the key tree.

In these situations, instead of removing $M$ (as well as its phantom location) from the key tree and recursively updating all the keys on its key path, the whole key tree will be reorganized to generate a new PF tree as the main tree, and the join tree is set to be empty. This will reduce the rekeying cost, as well as maintain a good key tree structure. The basic procedure is to first remove all the phantom nodes in the existing key tree and then apply the split procedure to partition the remaining key tree into many small full key trees that are indexed according to their size and their locations in the original key tree. After changing a certain member's secret share, the unite procedure will be applied to combine these full key trees into a PF key tree. Finally, all members will update the key tree structure maintained by themselves according the above procedure.

It is worth noting that due to the special structure of the PFMH tree, the PFMH tree structure is maintained after removing some phantom nodes. According to Procedure 4, Scenario 3, only those leaf nodes on the rightmost of the tree can be phantom nodes. In other words, all phantom nodes lie in the rightmost part of the tree. It is easy to check that for any PF tree, after removing any number of rightmost leaf nodes and those corresponding nonleaf nodes, the remaining part is still a PF tree.

Since all the remaining members (leaf nodes) know the exact structure of the key tree, after applying the split procedure, the set of obtained full key trees will be indexed in the same way by all group members. Since the total number of remaining members is less than $n$, according to Theorem 1, clause 1 , the total time cost is upper bounded by $\log n$. If situation 1,2 , or 3 happens, the total number of full key trees after applying the split procedure is upper bounded by $\log (n)+\left|T_{\text {join }}\right|$. In this case, the total communication cost in terms of multicast is upper bounded by $2\left(\log (n)+\left|T_{\text {join }}\right|\right) C_{\text {multicast }}$. If situation 4 happens, the total communication cost in terms of multicast is upper bounded by $2\left(2 \log n+\left|T_{\text {join }}\right|\right) C_{\text {multicast }}$, where the extra $2 \log n C_{\text {multicast }}$ is due to the fact that the main tree can be split into at most $2 \log n$ full trees.

Next, we analyze the computation cost under this scenario, which is mainly incurred by the unite procedure. After applying the split procedure, for any size that is greater than 1 , there exists no more than one full key tree with this size when situation 1,2, or 3 happens, and there exists no more than two full key trees with this size when situation 4 happens. The unite procedure can be implemented in two steps. In the first step, all the key trees with only one leaf node will first be combined together into a set of full key trees with different sizes. In the second step, these full key trees will be combined together with the other full key trees obtained by applying the split procedure to get the final PF tree. We first consider the more probable case that $T_{1} \geq n / 2$, where $T_{1}$ is the largest full key tree obtained after applying the split procedure. According to Theorem 1, clauses 3 and 5, in this case, the computation cost is upper bounded by $C_{m e}\left(2.5 n+2\left(\log n+\left|T_{\text {join }}\right|\right)+\left|T_{\text {join }}\right|\left(\log \left(\left|T_{\text {join }}\right|\right)+1\right)\right)$, where 


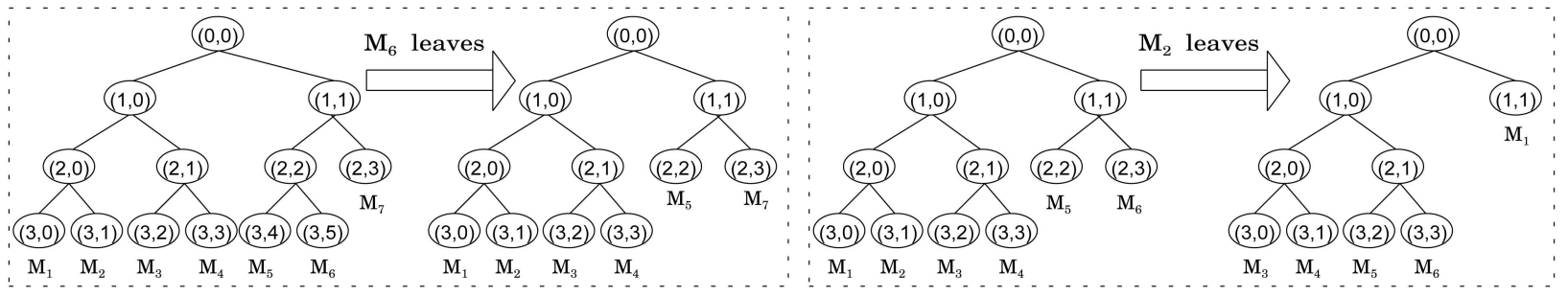

(a)

(b)

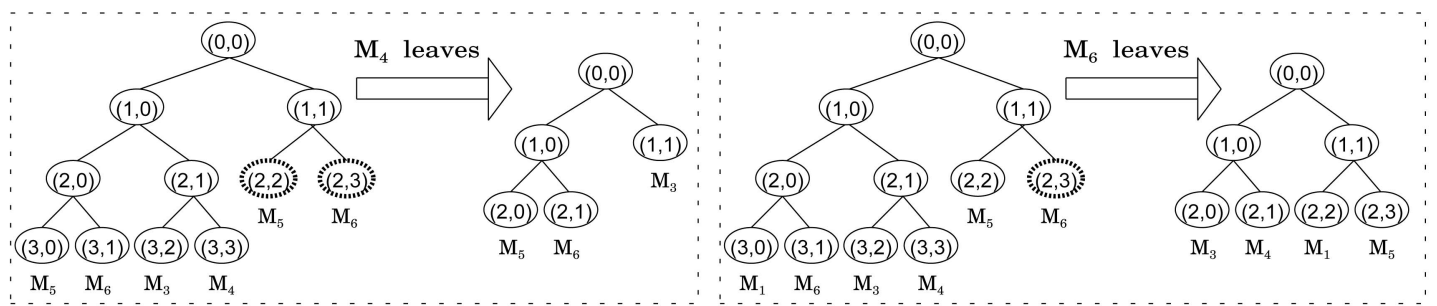

(c)

(d)

Fig. 6. Examples of a key tree update upon single-user leave under the second scenario. (a) Situation 1. (b) Situation 2 . (c) Situation 3. (d) Situation 4 .

the term $\left|T_{\text {join }}\right|\left(\log \left(\left|T_{\text {join }}\right|+1\right)\right)$ comes from merging the nodes from the join tree into a set of full key trees with different sizes. If $T_{1}<n / 2$, which is a less probable case, according to Theorem 1 , clauses 3 and 6 , the total computation cost is upper bounded by

$$
\left(3 n+2\left(\log n+\left|T_{\text {join }}\right|\right)+\left|T_{\text {join }}\right|\left(\log \left(\left|T_{\text {join }}\right|\right)+1\right)\right) C_{m e} .
$$

Similarly, the total communication cost in terms of unicast is upper bounded by $\left(2.5 n+\left|T_{\text {join }}\right| \log \left(\left|T_{\text {join }}\right|\right)\right) C_{\text {unicast }}$ if $T_{1} \geq$ $n / 2$ and is upper bounded by $\left(3 n+\left|T_{\text {join }}\right| \log \left(\left|T_{\text {join }}\right|\right)\right) C_{\text {unicast }}$ if $T_{1}<n / 2$.

If condition 4 is satisfied, which is a very rare event, at most $(n+\log n) C_{m e}$ extra computation cost is needed to first combine those full key trees with the same size into a set of larger full key trees, and at most $n C_{\text {unicast }}$ extra communication cost in terms of unicast is needed.

Fig. 6 shows four examples of a key tree update upon single-user leave under this scenario:

- The first example corresponds to situation 1. The leaving member $M_{6}$ is in the join tree, and the size of join tree with root $(1,1)$ is $\operatorname{larger}$ than $\log n$. In this example, after removing $M_{6}$ and applying the split procedure, three full key trees (subgroups) are obtained: $\left\{M_{1}, M_{2}, M_{3}, M_{4}\right\},\left\{M_{5}\right\}$, and $\left\{M_{7}\right\}$. The result of the unite procedure has also been demonstrated.

- The second example corresponds to situation 2. The leaving member $M_{2}$ is in the main tree with $\operatorname{root}(1,0)$, and the size of the join tree with root $(1,1)$ is larger than 1 . In this case, after removing $M_{2}$ and applying split, three full key trees are obtained: $\left\{M_{3}, M_{4}\right\}$, $\left\{M_{5}, M_{6}\right\}$, and $\left\{M_{1}\right\}$. The result of unite has also been illustrated in the right side of the figure.

- The third example corresponds to situation 3. The leaving member $M_{4}$ is in the main tree with root $(0,0)$ (the join tree is empty) and is the rightmost nonphantom leaf node, where nodes $(2,2)$ and $(2,3)$ are phantom nodes. In this case, after removing the node representing $M_{4}$ and the phantom nodes and applying split, two full key trees are obtained: $\left\{M_{5}, M_{6}\right\}$ and $\left\{M_{3}\right\}$. The result of unite has also been illustrated in the right side of the figure.

- The fourth example corresponds to situation 4. The leaving member $M_{6}$ is in the main tree with root $(0,0)$ (the join tree is empty) and has occupied a phantom node $(2,3)$. In this case after removing the node representing $M_{4}$ and the phantom node and applying split, three full key trees are obtained: $\left\{M_{3}, M_{4}\right\},\left\{M_{1}\right\}$, and $\left\{M_{5}\right\}$. The result of unite has also been illustrated in the right side of the figure.

SCENARIO 3. This scenario covers all the situations that neither of the first two scenarios can cover. Specifically, this scenario considers two situations: 1) $M$ is in the main tree, and the size of the join tree is 1, and 2) the join tree is empty, and $M$ is in the main tree, is not the rightmost nonphantom node, and does not have a phantom node in the key tree. Under Scenario 3, the leaving member $\mathrm{M}$ is removed from the key tree, and $M^{\prime}$, which is the member who occupies the rightmost nonphantom leaf node, moves to M's previous position, generates a secret share for this node, and recursively updates all the keys on this node's key path. Now, $M^{\prime}$ occupies two positions, and the original position is called $M^{\prime \prime}$ s phantom position. It is easy to check that the time cost is bounded by $\log n$, the communication cost in terms of multicast is bounded by $2(\log n) C_{\text {multicast }}$, the computation cost is upper bounded by $\left(n+2\left|T_{\text {left }}\right|+\log n\right) C_{m e}$, where $T_{\text {left }}$ is $T_{\text {main }}$ 's left subtree, and the total communication cost in terms of unicast is upper bounded by $\left(n+2\left|T_{\text {left }}\right|\right) C_{\text {unicast }}$.

Fig. 7 shows one example of a key tree update upon single-user leave under this scenario. In this example, the join tree is empty, and the root of the main tree is $(0,0)$. When user $M_{2}$ leaves the group, member $M_{6}$ will move to the location $(3,1)$ that previously represents $M_{2}$. Meanwhile, $M_{6}$ will also occupy node $(2,3)$, which now is a phantom node. $M_{6}$ will change its secret share and 


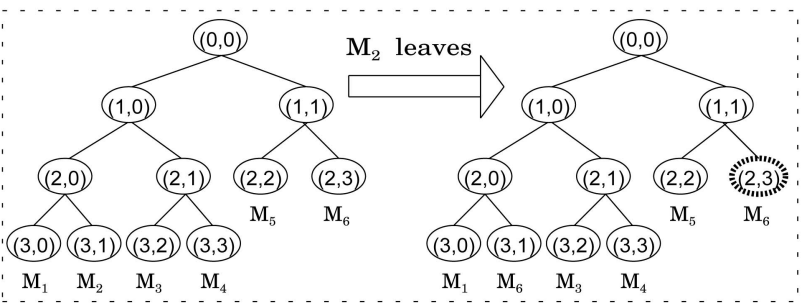

Fig. 7. An example of a key tree update upon single-user leave under the third scenario.

recursively update all the keys on its key path, which is $\{(2$, $0),(1,0),(0,0)\}$.

Table 2 summarizes the rekeying cost upon single-user leave events under different situations. Usually, we have $\left|T_{\text {left }}\right| \geq n / 2, h \simeq \frac{1}{2} \log n$, and $\left|T_{\text {join }}\right| \ll n$, and the average size of $T_{\text {left }}$ is about $0.75 n$. For the second and third scenarios, in most cases, we can simplify the upper bound of the computation cost as $O\left(2.5 n C_{m e}\right)$. For the first scenario, we can simplify the bound of the computation cost as $O\left(n C_{m e}\right)$.

\subsection{Group Merge and Group Partition Protocols}

PACK also has group merge and group partition protocols to handle simultaneously the join and leave of multiple users. Although multiple user events can be implemented by applying a sequence of single-user join or leave protocols, such sequential implementations are usually not cost-efficient. Procedure 5 describes the group merge protocol, which combines two or more groups into a single group, and returns a PF key tree. Procedure 6 describes the group partition protocol, which removes multiple group members simultaneously from the current group and construct a new PF key tree for the rest of the group members.

Procedure 5. merge $\left(\left\{\mathcal{G}_{1}, \ldots, \mathcal{G}_{K}\right\}\right)$

$\triangleright T_{1}, \ldots, T_{K}$ are the key trees of $\mathcal{G}_{1}, \ldots, \mathcal{G}_{K} ;$

Remove all phantom nodes from $T_{1}, \ldots, T_{K}$;

$T=$ unite $\left(\operatorname{split}\left(T_{1}\right) \bigcup \ldots \bigcup \operatorname{split}\left(T_{K}\right)\right)$;

Return $T$.

Procedure 6. Partition $\left(\mathcal{G}, \mathcal{G}_{1}\right)$

$\triangleright T$ is the key tree of $\mathcal{G}$;

Remove all phantom members and members belonging to group $\mathcal{G}_{1}$ from $T$;

$T=$ unite $(\operatorname{split}(\mathcal{T}))$;

Return $T$.
In the group merge protocols, after removing all phantom nodes from those key trees corresponding to different subgroups, each key tree is split into several full key trees. The final result is obtained by uniting these full key trees into a PF tree following Procedure 1. Similar to the group partition protocol, after removing all phantom nodes and leaving nodes, the original key tree is split into several full key trees, and the unite procedure is then applied on these full key trees to create a PF key tree. Since the height of the returned tree is $\log n$, where $n$ is the group size after merging/partitioning, the time cost of group merge/partition is bounded by $O(\log n)$. Obviously, the group merge and partition protocols have a lower cost than the sequential implementations.

\section{Performance Evaluation and Comparison}

\subsection{Forward and Backward Security}

Group key secrecy means that attackers cannot obtain the group key even if they know all blind keys, which has been proved in the random-oracle model [23]. To show that PACK satisfies forward and backward secrecy, similar arguments as in [9] can be used, which have provided a detailed proof for TGDH. PACK and TGDH use similar group key update procedures. The major difference between them is in the underlying key tree structures, which do not affect the security of the scheme. Therefore, in this paper, we will not provide a detailed proof of forward and backward secrecy. Next, we only roughly sketch the proof. We first consider backward secrecy. When a new user $\mathrm{M}$ wants to join the group, $\mathrm{M}$ picks its secret share $r$. After several rounds of the two-group $\mathrm{DH}, \mathrm{M}$ gets all blinded keys on its copath, and it can compute all secret keys on its key path using its own secret share and the blinded keys on its copath. Clearly, all of these keys contain M's secret share; hence, they are independent of the previous secret keys on that path. Therefore, $M$ cannot derive any previous keys. The forward secrecy can be shown in a similar way. When a member $M$ leaves the group, at least one current member changes its share, and all the keys on M's key path will be updated to remove M's secret share. Hence, M only knows at most all blinded keys, and the group key secrecy property prevents $\mathrm{M}$ from deriving any future group keys. By combining backward secrecy and forward secrecy, we can derive the key independence.

TABLE 2

Rekeying Cost Bounds upon a Single-User Leave Event

\begin{tabular}{|l|c|c|c|c|}
\hline & $\begin{array}{c}\text { time cost } \\
\text { (rounds) }\end{array}$ & $\begin{array}{c}\text { communication cost } \\
\left(C_{\text {multicast }}\right)\end{array}$ & $\begin{array}{c}\text { communication cost } \\
\left(C_{\text {unicast }}\right)\end{array}$ & $\begin{array}{c}\text { computation cost } \\
\left(C_{m e}\right)\end{array}$ \\
\hline Scenario 1 & $O(h)$ & $O(2 h-2)$ & $O\left(n+0.5\left|T_{\text {join }}\right|^{2}\right)$ & $O\left(n+0.5\left|T_{\text {join }}\right|^{2}\right)$ \\
\hline Scenario 2 & $O(\log n)$ & $O\left(2 \log n+2\left|T_{\text {join }}\right|\right)$ & $O\left(2.5 n+\left|T_{\text {join }}\right| \log \left(\left|T_{\text {join }}\right|\right)\right)$ & $O\left(2.5 n+\left|T_{\text {join }}\right| \log \left(\left|T_{\text {join }}\right|\right)\right)$ \\
\hline Scenario 3 & $O(\log n)$ & $O(2 \log n)$ & $O\left(n+2\left|T_{\text {left }}\right|\right)$ & $O\left(n+2\left|T_{\text {left }}\right|\right)$ \\
\hline
\end{tabular}


TABLE 3

Rekeying Cost Comparison among Different Schemes

\begin{tabular}{|c|c|c|c|}
\hline & time cost & communication cost & computation cost \\
\hline \multicolumn{3}{|c|}{ Upon Single User Join Event } \\
\hline PACK & $1 \sim 2$ & $2 \sim 4 C_{\text {multicast }}$ & $n C_{m e}$ \\
\hline TGDH & $\log n$ & $2(\log n) C_{m u l t i c a s t}$ & $2 n C_{m e}$ \\
\hline DST & $1+\log \log n$ & $(1+\log \log n) C_{m u l t i c a s t}$ & $(n+\log n) C_{m e}$ \\
\hline \multicolumn{4}{|c|}{ Upon Single User Leave Event } \\
\hline PACK & $\log n$ & $2(\log n) C_{m u l t i c a s t}$ & $(1 \sim 2.5) n C_{m e}$ \\
\hline TGDH & $\log n$ & $2(\log n) C_{m u l t i c a s t}$ & $2 n C_{m e}$ \\
\hline DST & $1+\log n+\log \log n$ & $2(1+\log n+\log \log n) C_{m u l t i c a s t}$ & $3 n C_{m e}$ \\
\hline
\end{tabular}

\subsection{Cost Comparison}

This section compares the rekeying cost in PACK upon single-user join and leave events with that of two existing tree-based contributory group key agreement schemes: TGDH [9] and DST [10]. All three types of costs are considered: time, computation, and communication in terms of multicast. Since, in general, a members' leaving time is not known in advance, in DST, only the join tree is used. Table 3 lists the approximate bounds of the different costs for the three schemes.

From the above comparison, we can see that PACK has the lowest cost in terms of time, computation, and communication. For example, for user join, only one or two rounds are needed in time cost, whereas DST needs $1+\log \log n$ rounds, and TGDH needs $\log n$ rounds. Similar results can also be seen in the communication cost for user join. The total computation cost is computed as the average of the user join cost and leave cost; DST has a similar cost as TGDH, which is an order of $2 n$, whereas for PACK, the order is from $n$ to $1.75 n$, with the savings ranging from 15 percent to 50 percent compared with that of DST and TGDH.

\subsection{Simulation Results}

In our simulations, we generate the user activities according to the following probabilistic models: Users join the group according to a Poisson process with the average arrival rate $\lambda$, and the users' staying time in the group follows an exponential distribution with mean $\mu$ (such a model is motivated by the user statistics in study of Mbone [24], [25]). Then, $\lambda \mu$ is the average number of users in the group, that is, the average group size. For each simulation, we initialize the group size to be 0 , fix $\lambda$, and vary $\mu$ to get different average group size configurations. For each configuration (different average group size), a sequence of $100 \lambda \mu$ users join the group according to the Poisson process with rate $\lambda$, and each user's staying time is drawn independently from an exponential distribution with mean $\mu$. In the simulations, we have compared the rekeying costs of the three schemes, PACK, TGDH [8], and DST [10], in all three aspects: computation, communication, and time.

The simulation results are presented in Fig. 8. From these results, we can see that upon a single-user join event, PACK has the lowest cost among all three schemes. Compared with DST, PACK has a more than 10 percent reduction in computation cost and a more than 65 percent reduction in communication cost and time cost. Compared with TGDH, the reduction is even more, about 50 percent in computation cost and about 80 percent in time and communication costs. Upon a single-user leave event, compared with DST, PACK has about a 25 percent reduction in computation cost, about a 15 percent reduction in time cost, and a similar communication cost. Although PACK has slightly higher computation and communication costs than TGDH upon a singleuser leave event, when averaged over both join and leave events, the reduction is still significant, with a 20 percent reduction in computation cost, 35 percent reduction in communication cost, and 40 percent reduction in time cost.

\section{Contributory Group Key Agreement with KeY VALIDATION}

In practice, there may exist malicious or compromised group members who do not perform the key agreement protocol honestly and cause key generation failure. One example of key generation failure is group partition where some users share one key while the others share another different key. Therefore, besides the four security requirements discussed in Section 2, the group key management should also have the key validity property. That is, without being detected by other users, malicious users cannot prevent a valid group key from being generated by providing false information. In this section, we discuss the possible damage that untruthful users can cause and the mechanisms to check the key validity.

When implementing the two-group $\mathrm{DH}$ using the method described in Section 2, an untruthful member can cause key generation failure only if it has been elected as a delegate. In this case, an untruthful member, for example, $a$ in subgroup $\mathbf{A}$, can send a false blinded key $f\left(K_{A}^{\prime}\right)$ to selected members in subgroup $\mathbf{B}$. As a consequence, those members in $\mathbf{B}$ who have received false blinded keys from $a$ cannot obtain the valid group key $K_{A B}$, that is, these members have been implicitly revoked from the new group.

We introduce two methods to check the validity of the key establishment procedure and to detect malicious members. One is preventive, and the other is detective. In the preventive scheme, for each group, $m$ members are elected as delegates and broadcast the blinded key. Then, each group member checks whether these $m$ copies of blinded keys are the same. Since all the keying messages 

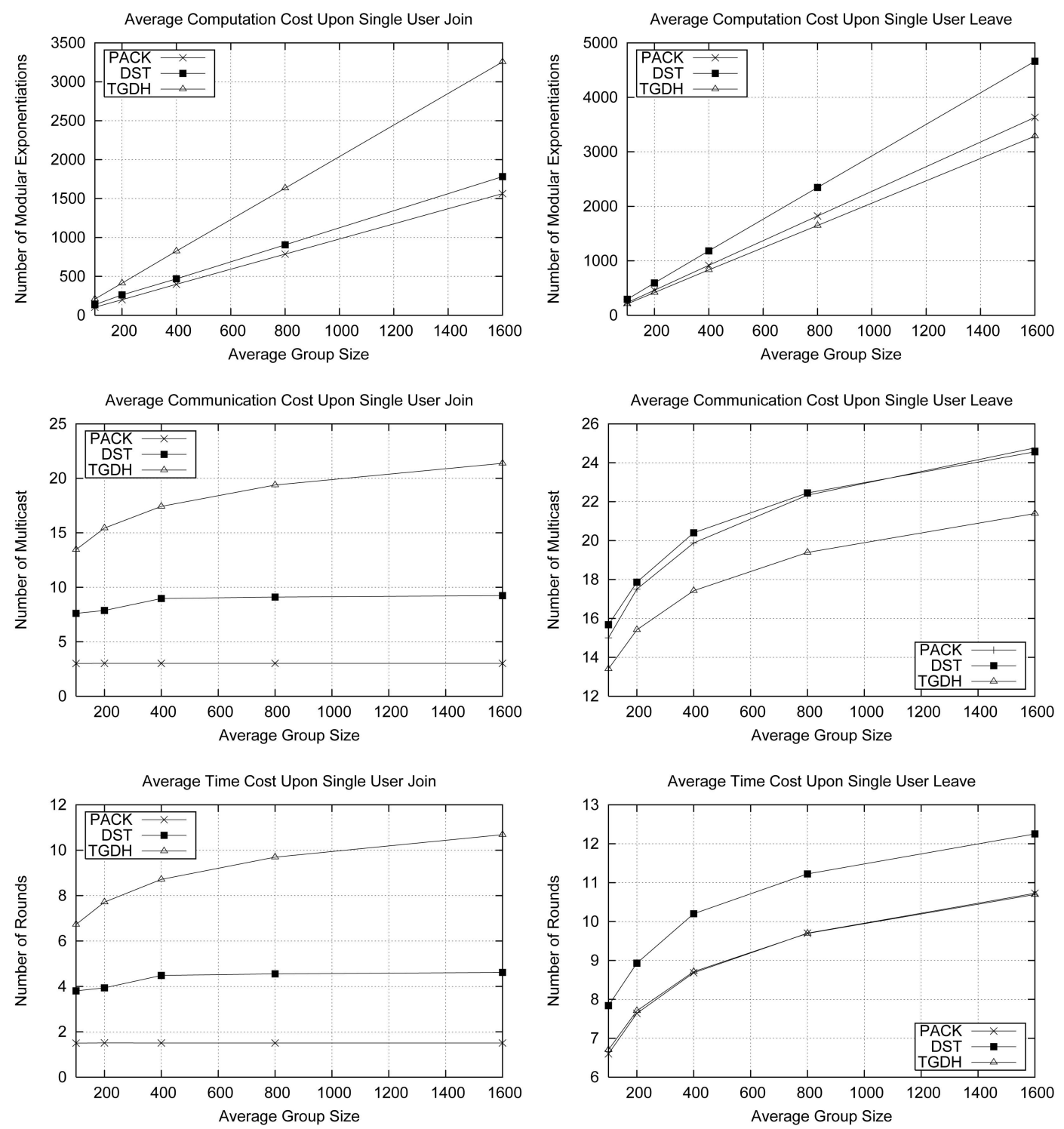

Fig. 8. Comparison of rekeying costs among PACK, TGDH, and DST.

have been signed by the senders, the member who has sent false information can be easily detected by other group members. In the detective scheme, after the each round of $\mathrm{DH}, m$ members are elected to broadcast a common known message encrypted using the newly generated group/ subgroup key. Other members check whether they can use their new group/subgroup key to successfully decrypt the message. If a user cannot obtain this commonly known message after decryption, it broadcasts an error message that includes the blinded key and the messages it has received. Again, since keying messages are signed by their senders, those malicious members who have sent a false blinded key or false encrypted messages can be detected.

Although colluders can compromise both preventive and detective schemes, the probability of a successful collusion attack is very low because those $m$ delegates or $m$ users who broadcast the encrypted message are randomly selected. In addition, the detective method is more resistant to collusion attacks than the preventive methods. In the preventive method, the $m$ delegates are selected within one

subgroup, whereas in the preventive method, the $m$ users are selected from both subgroups.

Key validation requires an extra cost. In each round of the two-group $\mathrm{DH}$, the preventive scheme requires $2 m$ broadcast, and the reactive scheme requires $m$ broadcast, $m$ encryption, and $n$ decryption, where $n$ is the size of the new subgroup after the DH round. It is noted that the extra cost due to checking is proportional to the cost of the key management schemes without the checking schemes. Thus, in the previous analysis and comparisons, we did not count the extra cost associated with key validation.

\section{Conclusion}

In this paper, we designed PACK, a highly efficient contributory key agreement scheme that has much lower communication, computation, and time overhead than existing schemes and achieves the performance lower bound derived in [22]. PACK reduces the overhead associated with key updating in two ways. First, it uses the novel PFMH tree 
structure that consists of a main tree, which is optimal for user leave, and a join tree, which is optimal for user join. Second, the concept of phantom user location in the PFMH allows the cost amortization when handling user leave. Upon single-user join, PACK has the time cost as one or two rounds of the two-group $\mathrm{DH}$, the communication cost as two or four multicast, and the average computation cost as one modular exponentiation per user. Upon a single-user leave event, PACK takes at most $\log n$ rounds of the two-group DH in terms of time cost, $O(\log n)$ multicast in communication cost, and an average of 2 modular exponentiations per user in computation cost, where $n$ is the current group size. The performance of PACK is compared with that of TGDH and DST. Both theoretical bound analysis and simulation results have shown that PACK has much lower rekeying costs in terms of communication, computation, and time than existing schemes.

\section{APPENDIX \\ ProOF OF THEOREM 1}

1. Consider the worst-case scenario: $L=n$, that is, $\left|T_{l}\right|=1$ for all $l$. Then, Procedure 1 works as follows: In the first round, the set of group members are partitioned into $\lceil n / 2\rceil$ subgroups, with each subgroup consisting of one or two members. For any subgroup of size 2, the two-group DH is performed between the two members in this subgroup to generate a new key tree of size 2 . In the $i$ th round, the set of existing key trees are partitioned into $\left\lceil n / 2^{i}\right\rceil$ subgroups, with each group consisting of one or two existing key trees. If there is a subgroup consisting of only one existing key tree, then this key tree must have the minimum size (largest index) among all the existing trees. For any subgroup with two existing key trees, the two-group $\mathrm{DH}$ is performed between these two key trees to generate a new key tree with its right child being the key tree that has a smaller size (larger index). Repeat this procedure until only one tree is left, which is the final PF key tree. Since there are only $n$ group members, at most $\log n$ rounds are needed, so the time cost is upper bounded by $\log n$. For other scenarios where there exists $\left|T_{i}\right| \neq 1$, the time cost is always no more than $\log n$, since in these cases $T_{i}$ can be viewed as the result of merging all the leaf nodes in $T_{i}$ without introducing any time cost.

2. Since we need and only need to perform $L-1$ times of two-group $\mathrm{DH}$ protocols to unite $L$ full key trees into one PF tree and since each two-group $\mathrm{DH}$ protocol needs two multicast in communication cost provided that the exchange of keying material between two subgroups during performing the two-group $\mathrm{DH}$ is implemented using multicast, the total communication cost is always upper bounded by $2(L-1) C_{\text {multicast }}$.

3. According to Procedure 1, we know that at most $\log n$ rounds of the two-group $\mathrm{DH}$ need to be performed in this situation. At the first round, each member calculates its blinded key and a new subgroup key. At the $i$ th round $(i>1)$, at most $\left\lceil n / 2^{i-1}\right\rceil$ users (which are selected as delegates) need

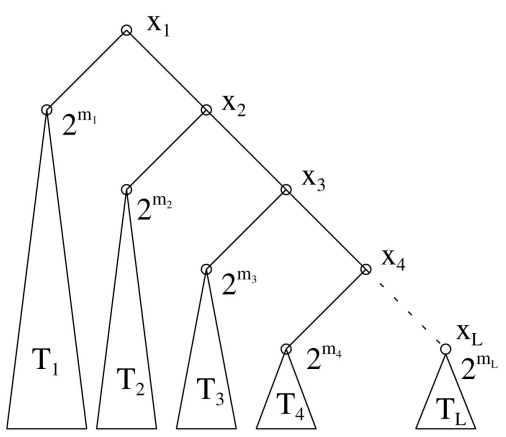

Fig. 9. Obtained PF key tree after applying the unite procedure.

to calculate their blinded keys, and at most $n$ users need to calculate their subgroup keys. Following this analysis, we can see that the total computation cost is upper bounded by $n(\log n+2) C_{m e}$. Further, if the exchange of keying material between two subgroups during performing the two-group $\mathrm{DH}$ is implemented using unicast, it is easy to see that a blinded key needs to send to a certain member if and only if this member needs to calculate a key for a newly generated subgroup that it belongs to, which is equivalent to say that the total communication cost is upper bounded by $(n \log n) C_{\text {unicast }}$ in this situation.

4. In this special situation, according to the definition of a PF tree, it is easy to check that the key tree illustrated in Fig. 9 is the obtained PF key tree after applying Procedure 1. Assume that the size of each full subtree $T_{i}$ is $2^{m_{i}}$, and let $x_{i}$ denote both the PF subtree and its size. According to Procedure 1, we know that the PF subtrees $x_{L}, \ldots, x_{1}$ are generated sequentially with $x_{L}$ first (directly from $\mathcal{T}$ ) and $x_{1}$ last. Also, when $x_{i}$ is generated, at most $x_{i}+2$ modular exponentiation operations are needed, so the total computation cost is upper bounded by

$$
C_{m e} \sum_{i=1}^{L}\left(x_{i}+2\right)=C_{m e}\left(2 \log n+\sum_{i=1}^{L} x_{i}\right) .
$$

Since we have

$$
\begin{gathered}
x_{i}=2^{m_{i}}+x_{i+1}, \\
x_{i} \geq 2 x_{i+1}, \\
\sum_{i=2}^{L} x_{i}<x_{1}=n,
\end{gathered}
$$

the above bound is further upper bounded by $2(n+\log n) C_{m e}$. Meanwhile, it is easy to check that the total communication cost is upper bounded by $2 n C_{\text {unicast }}$ in this situation provided that the exchange of keying material between two subgroups during performing the two-group $\mathrm{DH}$ is implemented using unicast.

5. In this special situation, let the key tree illustrated in Fig. 10a be the PF tree obtained after applying the unite procedure. Now, consider the computation cost incurred by the full subtree $T_{i}^{\prime}$ : 


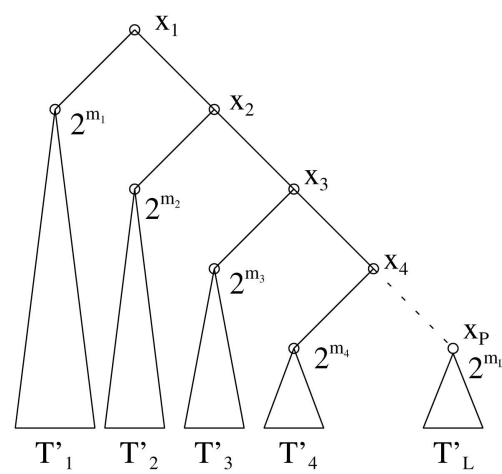

(a)

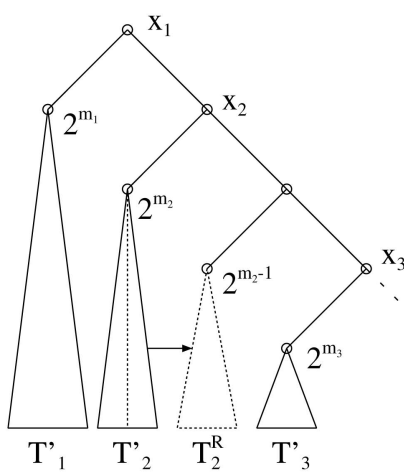

(b)

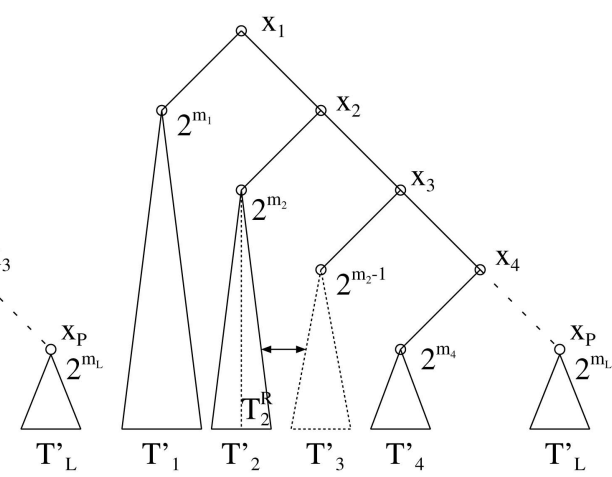

(c)

Fig. 10. Analysis of the computation cost. (a) Obtained PF key tree.

(b) Create a virtual subtree for $T_{2}^{R}$. (c) Exchange $T_{2}^{R}$ with $T_{3}^{\prime}$.

Case 1 . If $T_{i}^{\prime}$ comes directly from the original set $\mathcal{T}$, the cost introduced by $T_{i}^{\prime}$ has been totally included in (3).

Case 2. If $T_{i}^{\prime}$ is the merging result of two full trees directly from the original set $\mathcal{T}$ and each with size $2^{m_{i}-1}$, compared with the first case, an extra computation cost is needed to first merge the two trees into a single full tree. Since the total number of leaf nodes in $T_{i}^{\prime}$ is $2^{m_{i}}$ and each leaf node needs one modular exponentiation to calculate the new subgroup key associated to $T_{i}^{\prime}$, the extra computation cost introduced by $T_{i}^{\prime}$ is $2^{m_{i}} C_{m e}+2$.

Case 3. If $T_{i}^{\prime}$ is the merging result of more than two full key trees of the original set $\mathcal{T}$, since we have assumed that for each size, the number of trees with this size in $\mathcal{T}$ is no more than 2 , then at least one child of $T_{i}^{\prime}$ with size $2^{m_{i}-1}$ comes directly from $\mathcal{T}$. Let $T_{i}^{L}$ and $T_{i}^{R}$ be the left and right children of $T_{I}^{\prime}$, and assume that $T_{i}^{L}$ comes directly from $\mathcal{T}$. In this case, either there exists no key tree with size $2^{m_{i}-1}$ in the right side of $T_{i}^{\prime}$ or if there exists $\left|T_{i+1}^{\prime}\right|=2^{m_{i}-1}$, then $T_{i+1}^{\prime}$ must come directly from $\mathcal{T}$ in order not to violate the assumption that no more than two key trees in $\mathcal{T}$ have the same size, and $T_{i+1}^{\prime}$ will not introduce an extra cost except those included in (3). If there exists no subtree with size $2^{m_{i}-1}$ in the right side of $T_{i}^{\prime}$, we add a virtual subtree $T_{i}^{R}$ to the generated PF tree as in Fig. 10b and move all the cost introduced by merging smaller full trees into this subtree. If $\left|T_{i+1}^{\prime}\right|=2^{m_{i}-1}$, we can simply exchange the subtree $T_{i+1}^{\prime}$ with the right subtree $T_{i}^{R}$ of $T_{i}^{\prime}$ that is not directly from the original set $\mathcal{T}$, as in Fig. 10c. Now, the total cost is kept to be the same, but the extra cost introduced by $T_{i}^{\prime}$ is the same as in Case 2.

Following the above analysis and the condition that $\left|T_{1}\right| \geq n / 2$ (that is, $T_{1}$ comes directly from $\mathcal{T}$ ), the total extra computation cost that are not included in (3) is upper bounded by $\sum_{i=1}^{m_{2}} 2^{i} C_{m e}$. Now, the total computation cost is upper bounded by

$$
2 L C_{m e}+C_{m e}\left(\sum_{i=1}^{m_{2}} 2^{i}+\sum_{i=1}^{L} x_{i}\right) .
$$

By applying (4), (5), (6), and $\left|T_{1}\right|=2^{m_{1}} \geq n$, we have

$$
\begin{aligned}
\sum_{i=1}^{m_{2}} 2^{i} & +\sum_{i=1}^{L} x_{i} \leq 2^{m_{2}+1}+2 x_{1} \leq x_{1} / 2 \\
& +2 x_{1}=2.5 x_{1}=2.5 n
\end{aligned}
$$

That is, the total computation cost is upper bounded by $(2.5 n+2 L) C_{m e}$. Meanwhile, we can conclude that in this situation, when the exchange of keying materials is implemented using unicast, the total communication cost is upper bounded by $2.5 n C_{\text {unicast }}$.

6. For the special situation that $\left|T_{1}\right|<n / 2$ and for each tree $T_{i} \in \mathcal{T}$, there exists no more than one other tree in $\mathcal{T}$ with the same size as $T_{i}$, by following the same analysis as in (5), we can show that the total computation cost is upper bounded by

$$
2 L C_{m e}+C_{m e}\left(\sum_{i=1}^{m_{1}} 2^{i}+\sum_{i=1}^{L} x_{i}\right),
$$

where the only change from (7) to (9) is that $m_{2}$ is changed to $m_{1}$ due to the reason that $T_{1}^{\prime}$ does not come directly from $\mathcal{T}$.

By applying (4), we have that

$$
\begin{aligned}
\sum_{i=1}^{m_{1}} 2^{i} & +\sum_{i=1}^{L} x_{i}=x_{1}+\left(x_{2}+2^{m_{1}}\right)+\sum_{i=1}^{m_{2}} 2^{i} \\
+ & \sum_{i=3}^{L} x_{i} \leq 2 x_{1}+2^{m_{2}+1}+x_{2} \leq 3 x_{1}=3 n .
\end{aligned}
$$

That is, the total computation cost is upper bounded by $(3 n+2 L) C_{m e}$. Meanwhile, we can conclude that in this situation, when the exchange of keying materials is implemented using unicast, the total communication cost is upper bounded by $3 n C_{\text {unicast }}$.

\section{ACKNOWLEDGMENTS}

This work was supported in part by the US Army Research Office under URI Award No. DAAD19-01-1-0494. 


\section{REFERENCES}

[1] I. Ingemarsson, D.T. Tang, and C.K. Wong, “A Conference on Key Distribution System," IEEE Trans. Information Theory, vol. 28, no. 5, pp. 714-720, Sept. 1982.

[2] D.G. Steer, L. Strawczynski, W. Diffie, and M. Wiener, "A Secure Audio Teleconference System," Proc. Advances in Cryptology, pp. $520-528,1990$.

[3] M. Burmester and Y. Desmedt, "A Secure and Efficient Conference Key Distribution Scheme," Advances in Cryptology—Proc. Workshop Theory and Application of Cryptographic Techniques (EUROCRYPT'94), pp. 275-286, 1994.

[4] M. Steiner, G. Tsudik, and M. Waidner, "Diffie-Hellman Key Distribution Extended to Group Communication," Proc. Third ACM Conf. Computer and Comm. Security (CCS '96), pp. 31-37, 1996.

[5] K. Becker and U. Wille, "Communication Complexity of Group Key Distribution," Proc. Fifth ACM Conf. Computer and Comm. Security (CCS '98), pp. 1-6, 1998.

[6] G. Ateniese, M. Steiner, and G. Tsudik, "Authenticated Group Key Agreement and Friends," Proc. Fifth ACM Conf. Computer and Comm. Security (CCS '98), pp. 17-26, 1998.

[7] M. Steiner, G. Tsudik, and M. Waidner, "Key Agreement in Dynamic Peer Groups," IEEE Trans. Parallel and Distributed Systems, vol. 11, no. 8, pp. 769-780, Aug. 2000.

[8] Y. Kim, A. Perrig, and G. Tsukid, "Simple and Fault-Tolerant Key Agreement for Dynamic Collaborative Groups," Proc. Seventh ACM Conf. Computer and Comm. Security (CCS '00), May 2000.

[9] Y. Kim, A. Perrig, and G. Tsudik, "Tree-Based Group Key Agreement," ACM Trans. Information and System Security, vol. 7, no. 1, pp. 60-96, Feb. 2004.

[10] Y. Mao, Y. Sun, M. Wu, and K.J.R. Liu, "Dynamic Join-Exit Amortization and Scheduling for Time-Efficient Group Key Agreement," Proc. INFOCOM '04, 2004.

[11] Y. Amir, Y. Kim, C. Nita-Rotaru, J.L. Schultz, J. Stanton, and G. Tsudik, "Secure Group Communication Using Robust Contributory Key Agreement," IEEE Trans. Parallel and Distributed Systems, vol. 15, no. 5, pp. 468-480, May 2004.

[12] G.H. Chiou and W.T. Chen, "Secure Broadcasting Using the Secure Lock," IEEE Trans. Software Eng., vol. 15, pp. 929-934, Aug. 1989.

[13] S. Mittra, "Iolus: A Framework for Scalable Secure Multicasting," Proc. ACM Conf. Applications, Technologies, Architectures, and Protocols for Computer Comm. (SIGCOMM '97), pp. 277-288, 1997.

[14] C.K. Wong, M. Gouda, and S.S. Lam, "Secure Group Communications Using Key Graphs," Proc. ACM Conf. Applications, Technologies, Architectures, and Protocols for Computer Comm. (SIGCOMM '98), Sept. 1998.

[15] D.M. Wallner, E.J. Harder, and R.C. Agee, Key Management for Multicast: Issues and Architectures, Internet draft, work in progress, Sept. 1998.

[16] M.J. Moyer, J.R. Rao, and P. Rohatgi, "A Survey of Security Issues in Multicast Communications," IEEE Network, vol. 13, no. 6, pp. 12-23, Nov./Dec. 1999.

[17] M. Waldvogel, G. Caronni, D. Sun, N. Weiler, and B. Plattner, "The VersaKey Framework: Versatile Group Key Management," IEEE J. Selected Areas in Comm., vol. 17, no. 9, pp. 1614-1631, Sept. 1999.

[18] L.R. Dondeti, S. Mukherjee, and A. Samal, "DISEC: A Distributed Framework for Scalable Secure Many-to-Many Communication," Proc. Fifth IEEE Symp. Computers and Comm. (ISCC '00), pp. 693698, 2000.

[19] A. Perrig, D. Song, and D. Tygar, "ELK, A New Protocol for Efficient Large-Group Key Distribution," Proc. IEEE Symp. Security and Privacy, pp. 247-262, 2001.

[20] Y. Sun, W. Trappe, and K.J.R. Liu, "A Scalable Multicast Key Management Scheme for Heterogeneous Wireless Networks," IEEE/ACM Trans. Networking, vol. 12, no. 4, pp. 653-666, Aug. 2004.

[21] W. Diffie and M. Hellman, "New Directions in Cryptography," IEEE Trans. Information Theory, vol. IT-22, no. 6, pp. 644-654, Nov. 1976.

[22] J. Snoeyink, S. Suri, and G. Varghese, "A Lower Bound for Multicast Key Distribution," Proc. INFOCOM '01, 2001.

[23] M. Bellare and P. Rogaway, "Random Oracles Are Practical: A Paradigm for Designing Efficient Protocols," Proc. First ACM Conf. Computer and Comm. Security (CCS '93), 1993.
[24] K.C. Almeroth and M.H. Ammar, "Multicast Group Behavior in the Internet's Multicast Backbone (mbone)," IEEE Comm. Magazine, pp. 124-129, June 1977.

[25] K.C. Almeroth, "A Long-Term Analysis of Growth and Usage Patterns in the Multicast Backbone (mbone)," Proc. INFOCOM '00, vol. 2, pp. 824-833, Mar. 2000.

[26] Y. Mao, Y. Sun, M. Wu, and K.J.R. Liu, “JET: Dynamic Join-ExitTree Amortization and Scheduling for Contributory Key Management," IEEE/ACM Trans. Networking, vol 14, no 5, pp.1128-1140, Oct. 2006.

[27] W. Trappe, Y. Wang, and K.J.R. Liu, "Resource-Aware Conference Key Establishment for Heterogeneous Networks," IEEE/ACM Trans. Networking, vol 13, no 1, pp.134-146, Feb. 2005.

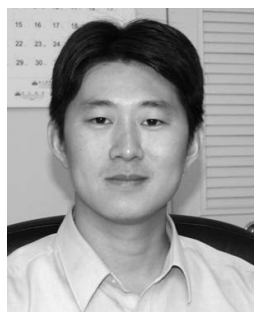

Wei $\mathrm{Yu}$ received the BS degree in computer science from the University of Science and Technology of China (USTC) in 2000, the MS degree in computer science from Washington University in St. Louis in 2002, and the PhD degree in electrical engineering from the University of Maryland in 2006. From August 2000 to May 2002, he was a graduate research assistant at Washington University in St. Louis. From September 2002 to July 2006, he was a graduate research assistant with the Communications and Signal Processing Laboratory and the Institute for Systems Research, University of Maryland. He joined Microsoft in August 2006. His research interests include network security, wireless communications, and networking, game theory, wireless multimedia, and pattern recognition.

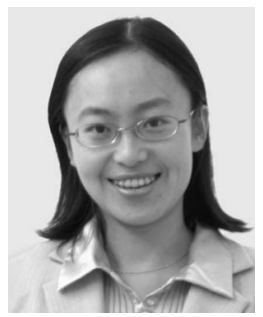

Yan (Lindsay) Sun received the BS degree with the highest honor from Beijing University, Beijing, in 1998 and the $\mathrm{PhD}$ degree in electrical and computer engineering from the University of Maryland in 2004. She is currently an assistant professor in the Electrical and Computer Engineering Department, University of Rhode Island. Her research interests include network security and wireless communications and networking. She received the Graduate School Fellowship at the University of Maryland from 1998 to 1999 and the Excellent Graduate Award of Beijing University in 1998. She received a US National Science Foundation (NSF) Faculty Early Career Development (CAREER) Award in 2007. She is a member of the IEEE, the IEEE Signal Processing Society, and the IEEE Communication Society.

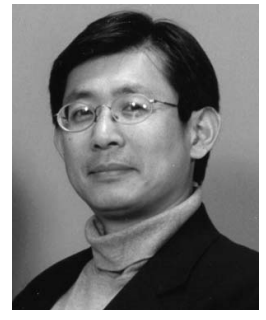

K.J. Ray Liu (F'03) received the BS degree from the National Taiwan University and the PhD degree from the University of California, Los Angeles (UCLA), both in electrical engineering. $\mathrm{He}$ is a professor and the associate chair of Graduate Studies and Research in the Electrical and Computer Engineering Department, University of Maryland, College Park. His research contributions encompass broad aspects of wireless communications and networking, information forensics and security, multimedia communications and signal processing, bioinformatics and biomedical imaging, and signal processing algorithms and architectures. He is the recipient of numerous honors and awards including best paper awards from the IEEE Signal Processing Society (twice), IEEE Vehicular Technology Society, and EURASIP; IEEE Signal Processing Society Distinguished Lecturer, EURASIP Meritorious Service Award, and the US National Science Foundation Young Investigator Award. He also received various teaching and research recognitions from the University of Maryland including the Distinguished Scholar-Teacher Award, Poole and Kent Co. Senior Faculty Teaching Award, and Invention of the Year Award. He is vice president-Publications and on the board of governors of the IEEE Signal Processing Society. He was the editor-in-chief of the IEEE Signal Processing Magazine and the founding editor in chief of the EURASIP Journal on Applied Signal Processing. He is a fellow of the IEEE.

$\triangleright$ For more information on this or any other computing topic, please visit our Digital Library at www.computer.org/publications/dlib. 